\title{
Different Approaches to Modelling the Cost-effectiveness of Schistosomiasis Control
}

\author{
Helen Guyatt \\ The Wellcome Trust Centre for the Epidemiology of Infectious Disease, Department of Zoology, University of \\ Oxford, South Parks Road, Oxford OX1 3PS, UK
}

This paper reviews three different approaches to modelling the cost-effectiveness of schistosomiasis control. Although these approaches vary in their assessment of costs, the major focus of the paper is on the evaluation of effectiveness. The first model presented is a static economic model which assesses effectiveness in terms of the proportion of cases cured. This model is important in highlighting that the optimal choice of chemotherapy regime depends critically on the level of budget constraint, the unit costs of screening and treatment, the rates of compliance with screening and chemotherapy and the prevalence of infection. The limitations of this approach is that it models the cost-effectiveness of only one cycle of treatment, and effectiveness reflects only the immediate impact of treatment. The second model presented is a prevalence-based dynamic model which links prevalence rates from one year to the next, and assesses effectiveness as the proportion of cases prevented. This model was important as it introduced the concept of measuring the long-term impact of control by using a transmission model which can assess reduction in infection through time, but is limited to assessing the impact only on the prevalence of infection. The third approach presented is a theoretical framework which describes the dynamic relationships between infection and morbidity, and which assesses effectiveness in terms of case-years prevented of infection and morbidity. The use of this model in assessing the cost-effectiveness of age-targeted treatment in controlling Schistosoma mansoni is explored in detail, with respect to varying frequencies of treatment and the interaction between drug price and drug efficacy.

Key words: schistosomiasis - mathematical modelling - cost-effectiveness analysis - control - morbidity

The resources available for investing in control programmes are limited. This necessities that consideration is given not only to the impact of a control programme, but also to the costs involved, both in terms of finances and operational requirements. There are a number of economic evaluation techniques which can be used to aid the decision making process in allocating scarce resources to schistosomiasis control. The techniques differ in the outcome measure assessed, and the choice of technique depends on the aims of the analysis. Cost-benefit analysis values the outcome in monetary terms and is concerned with the broad question of whether control of schistosomiasis is worthwhile in comparison with expenditure on other projects (health or otherwise). Cost-benefit analysis is difficult to apply to schistosomiasis control because of the complexities associated with valu-

This work received financial support from the Wellcome Trust.

Fax: +44-1865-281.245

E-mail: helen.guyatt@zoology.oxford.ac.uk

Received 4 May 1998

Accepted 31 August 1998 ing the health benefits. Cost-effectiveness analysis is concerned with identifying the most efficient option for controlling schistosomiasis among a range of alternative strategies. Cost-effectiveness analysis can be used to facilitate control programme design, once it is accepted that control of schistosomiasis is socially worthwhile.

The design of any schistosomiasis control programme involves the selection of one of a number of alternative interventions or a combination of several of them: control of the snail vector, improved water supplies or sanitation; or chemotherapy. Despite this diversity, most cost-effectiveness analyses have focused on strategies for delivering chemotherapy, as this is generally considered to be the most cost-effective approach to controlling schistosomiasis.

The choice of any strategy will ultimately depend on the cost-effectiveness ratio (cost per unit of effectiveness achieved) and existing budget constraints. The cost-effectiveness ratio may in turn be affected by a wide variety of economic, epidemiological, demographic, technical and behavioural factors. In the design of schistosome chemotherapy programmes these factors may include: compliance of the target population, drug efficacy, sensitivity of the screening test, preva- 
lence and intensity of infection, age-structure of the population, and the costs for screening, delivery and drugs. The analytical frameworks of many cost-effectiveness studies rarely consider all these factors, yet analysis has shown that the optimal choice of strategy can be extremely sensitive to variations in these.

In this paper examples of one static model and two dynamic models to assessing the cost-effectiveness of schistosomiasis control will be reviewed. These models address a number of delivery issues including the target population, mass treatment versus treatment based on diagnosis (screening), variations in the frequency of drug delivery, drug efficacy and drug price. The examples are theoretical, though in most cases based on empirical data on costs and representative data on epidemiological and behavioural parameters. The examples given differ in their approach to both cost and effectiveness evaluation. The choice of effectiveness measure is critical to the evaluation procedure, and is limited by the type of modelling approach employed. The limitations of static models are that effectiveness measures are usually simple immediate outcomes which reflect coverage (e.g. proportion of infected individuals treated) or both coverage and cure rate (e.g. proportion of infected individuals cured). The advantages of the dynamic models are that they can monitor changes in the parasite population over time in response to intervention, and can therefore monitor cases that were prevented because of intervention.

\section{STATIC MODEL}

Prescott (1987) developed a generalised static economic framework for investigating the optimal strategy for schistosomiasis chemotherapy. Prescott investigated the optimal treatment regime from a selection of four main strategies, variants on the target population (population or selected high-risk group) and prior diagnosis (mass or screening). His analysis demonstrated that the optimal choice of chemotherapy regime depends critically on the level of budget constraint, the unit costs of screening and treatment, the rates of compliance with screening and chemotherapy and the prevalence of infection. The objective of the intervention was to maximise the number of cases cured, and effectiveness was therefore assessed as the proportion of cases cured. The economic parameters consisted simply of a unit cost for treatment (2.50), and a unit cost for screening (0.50). The behavioural parameters were compliance with the mass chemotherapy alone (0.90), compliance with screening (0.75) and compliance with chemotherapy after screening $(0.95)$. These were assumed to be identical for the population and the selected high-risk group. The epidemiological parameters included the prevalence of infection in the population eligible for treatment $(50 \%)$ and the prevalence in the selected high-risk group (70\%). The effects of the interventions were further modified by the sensitivity of the screening test $(90 \%)$, and the efficacy of the drug $(90 \%)$. The costs and effectiveness of each approach were assessed simply as linear functions of the above parameters.

Prescott investigated the optimal choice of strategy with and without a budget constraint, and presented decision inequalities which must be satisfied if mass population chemotherapy is to be preferred over the other three strategies. For instance, in the absence of a budget constraint, mass population treatment will produce a greater proportion of cases cured than selective population chemotherapy if the compliance rate for mass treatment exceeds the compliance rate for screening weighted both by the sensitivity of the screening test and the compliance with treatment among screen-positives.

The optimal choice of chemotherapy regime was shown to be very sensitive to the level of budget constraint. The regimes targeted at a selected high-risk group tended to be more effective at the lower budget levels, but less effective at higher budget levels because they quickly exhaust their inherently limited capacity to cure cases. The screening regimes tended to be less effective than their mass counterparts because at any given budget level the fixed cost of screening has to be overcome before residual resources can be devoted to treating screen-positives. Prescott also examined the sensitivity of the choice of strategy to variations in the prevalence of infection and the unit cost of the screening test. He constructed an indifference line equation which defines for each unit cost per treatment, the combination of prevalence rates and screening costs for which planners would be indifferent between mass population chemotherapy and screening population chemotherapy (Fig. 1). In general, the lower the screening cost, the higher the prevalence rate required to justify mass treatment.

The analysis by Prescott (1987) models the cost-effectiveness of interventions applied for one year. Evaluation of a control programme implemented continuously for several years would require a transmission model linking prevalence rates from one year to the next, and this considered in the next section.

\section{DYNAMIC MODELS}

Prevalence-based dynamic model - Rosenfield et al. (1977) used a prevalence-based model of schistosomiasis transmission to simulate the effectiveness of different control strategies in Iran over 


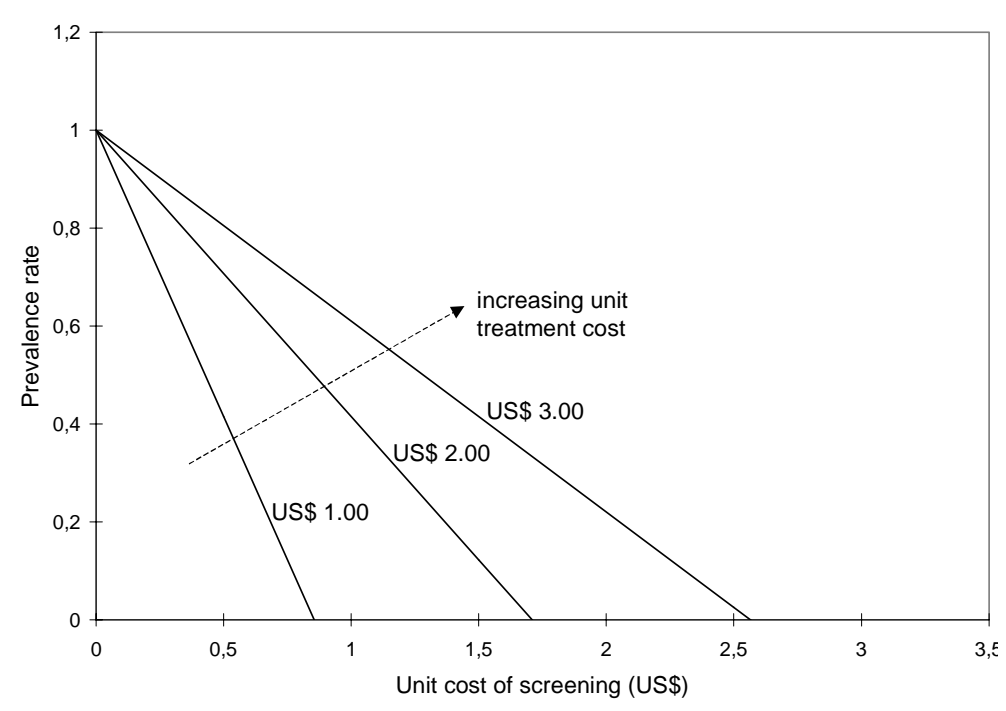

Fig. 1: the trade-offs between prevalence and screening costs in comparing mass chemotherapy with selective chemotherapy at different treatment costs. Constructed from indifference line by Prescott (1987): prevalence $=1$ - (unit screening cost/[unit treatment cost * compliance rate for selective chemotherapy $(0.95) *$ sensitivity of screening test $(0.90)])$.

a seven year period. The options were mollusciciding, engineering methods, chemotherapy, and a combination of these approaches. Maximum output in terms of the reduction in the prevalence of infection by the end of the seven year period was achieved with a combination of chemotherapy and mollusciciding. This intervention reduced the prevalence of infection from $64 \%$ to $20 \%$ whereas the next best alternative, chemotherapy, achieved a terminal prevalence of $60 \%$. Expressing effectiveness more appropriately in terms of the total number of cases prevented over this time period changed the ranking of alternatives. Chemotherapy yielded the greatest output at a cost per case prevented of US $\$ 1.26$, followed by the combination of controls with a cost-effectiveness ratio of US\$1.29 (Prescott \& Warford 1985).

Rosenfield's model was important as it introduced the concept of measuring the long-term impact of control by using a transmission model which can assess reduction in infection through time. However, the simulation relied on fitting the model to existing epidemiological data, and therefore limited its applicability to other endemic settings where such data is not always available. Furthermore, such data would be expensive and require a long time frame to collect, the latter also rendering it unsuitable for health planning. The model was also prevalence-based, and thus cannot give any indication of the reduction in disease. An alternative approach has been to model the impact on morbidity, and this is described in the next section.

Morbidity-based dynamic model - Chan et al. (1996a, b) have developed a theoretical framework which describes the dynamic relationships between infection and morbidity for schistosomiasis. In the model, infection intensity is modelled as a continuous function of age and time using a partial differential equation model. Morbidity is assessed at three levels, that due to current heavy infection, and early and late stages of chronic disease. The stages of chronic disease are modelled as a progression such that early disease develops in a manner related to the accumulated past experience of infection and late disease develops from early disease. Both types of morbidity are assumed to resolve spontaneously, although at different rates (early chronic disease at a greater rate than late chronic disease). Acquired immunity has also been incorporated into this framework (Chan et al. 1996b).

The rest of this paper will concentrate on the use of this dynamic model in investigating the costeffectiveness of age-targeted treatment (6-15 year olds) in the control of Schistosoma mansoni.

The model - Morbidity due to S. mansoni is divided into early chronic disease (hepatomegaly) and late chronic disease (Symmers fibrosis). Age targeted chemotherapy is simulated as an instantaneous reduction of infection intensity in the targeted age groups determined by coverage and drug efficacy. There will be two effects of treatment, the reduction of infection intensity in the treated age groups due to treatment, and the reduction in the other age groups due to the overall reduction in transmission. The age-structure of the popula- 
tion is based on an standard age curve for Africa (population $\mathrm{p}(\mathrm{a})=0.0455 . \mathrm{e}^{-(0.045 \mathrm{a})}$, where $\mathrm{p}(\mathrm{a})$ represents the density function or the proportion of people of age $a$ ) (Chan et al. 1995).

The inputs for the model include biological information on schistosomiasis (egg production in stool and adult worm lifespan), epidemiological information on schistosomiasis transmission (aggregation parameters, peak age of water contact, mean intensity at peak), development and resolution of disease, strength and duration of natural immunity, and factors related to the design of the programme (e.g. target age-group, frequency of treatment) and its effect (e.g. drug efficacy, coverage). The inputs for the model related to biology, disease, epidemiology and immunity, and the parameter values are given in Table I. Drug efficacy corresponds to the probability of worms being killed or the egg reduction rate.

Evaluating effectiveness - The outputs of the model are the prevalence of infection, prevalence of heavy infection (>250 e.p.g.), mean intensity of infection (average e.p.g.), prevalence of early disease (hepatomegaly) and prevalence of late disease (fibrosis) for each age (1-80) at each year for as many years as is specified. Fig. 2 illustrates the age profile of infection and morbidity at equilibrium (before treatment). Both infection intensity and hepatomegaly reach a peak at about age 15 . Symmers fibrosis remains at low levels, reaching a peak at about 25 years. At equilibrium, the mean e.p.g. is 144.00 , and prevalence of infection is $45.1 \%$, heavy infection is $14.5 \%$, hepatomegaly is $14.5 \%$ and fibrosis is $1.74 \%$. To illustrate the effect of treatment, let us consider the impact of treating 6-15 year olds every year for five years (in the first month of each year) with a drug of $90 \%$ efficacy and achieving an $80 \%$ coverage at each treatment cycle. Fig. 3 illustrates the changes in infection and morbidity over 15 years, where levels of infection and morbidity are presented relative to the equilibrium value. The greatest impact is on the prevalence of heavy infection and the mean intensity of infection which is reduced to approximately $20 \%$ of the equilibrium value at the end of the treatment programme. However, after the last treatment year, the prevalence and intensity of infection start to increase again, though at a lower rate than that induced by treatment. The prevalence of hepatomegaly continues to decrease for another two years, before an increase is seen. In sharp contrast, although the prevalence of fibrosis does not decrease at such a rapid rate as the other measures of infection and morbidity, it continues to decrease over the 15 year time horizon. The observed pattern for fibrosis is due to the low rates of development and resolution of this condition, and to the fact that the benefit in reducing the development of fibrosis in treated children is not observed until the children become adults (Chan \& Bundy 1997). The time horizon for evaluating the impact of the programme is clearly critical. If effectiveness, expressed as the percentage reduction in infection and morbidity, is assessed at year 5 , although this would capture the maximal effect on infection and heavy infection, it would greatly underestimate the impact on fibrosis.

The reduction in point prevalence of infection and morbidity at any time point does not capture the full effect of the treatment programme. An alternative, and preferred measure of effectiveness would be to look at cases-years prevented. In this

TABLE I

Parameter values used in the dynamic model for Schistosoma mansoni (Chan et al. 1995, 1996a, b)

\begin{tabular}{|c|c|c|}
\hline & Parameter & Value \\
\hline Biological & $\begin{array}{l}\text { Egg production in stool } \\
\text { Adult worm life span }\end{array}$ & $\begin{array}{l}5.26 \text { epg per worm } \\
4 \text { years }\end{array}$ \\
\hline Epidemiological & $\begin{array}{l}\text { Aggregation parameter (intercept) } \\
\text { Aggregation parameter (slope) } \\
\text { Peak age of water contact } \\
\text { Mean e.p.g. (arithmetic) at peak }\end{array}$ & $\begin{array}{l}0.132 \\
0.002 \\
15 \text { years old } \\
300\end{array}$ \\
\hline Disease & $\begin{array}{l}\text { Development rate for early disease }{ }^{a} \\
\text { Resolution time for early disease }{ }^{b} \\
\text { Development rate for late disease }{ }^{c} \\
\text { Resolution time for late disease }{ }^{b} \\
\text { Threshold e.p.g. for heavy infection }\end{array}$ & $\begin{array}{l}0.0071 \\
1 \text { year } \\
0.015 \\
13 \text { years } \\
250\end{array}$ \\
\hline Immunity & $\begin{array}{l}\text { Strength of immunity } d \\
\text { Duration of immunity } b\end{array}$ & $\begin{array}{l}42 \% \\
5 \text { years }\end{array}$ \\
\hline
\end{tabular}

$a$ : units: per worm per year; $b$ : rate $=1 /$ resolution time or duration; $c$ : units: per case of hepatomegaly per year; $d$ : percentage reduction in mean worm burden at age 20 . 


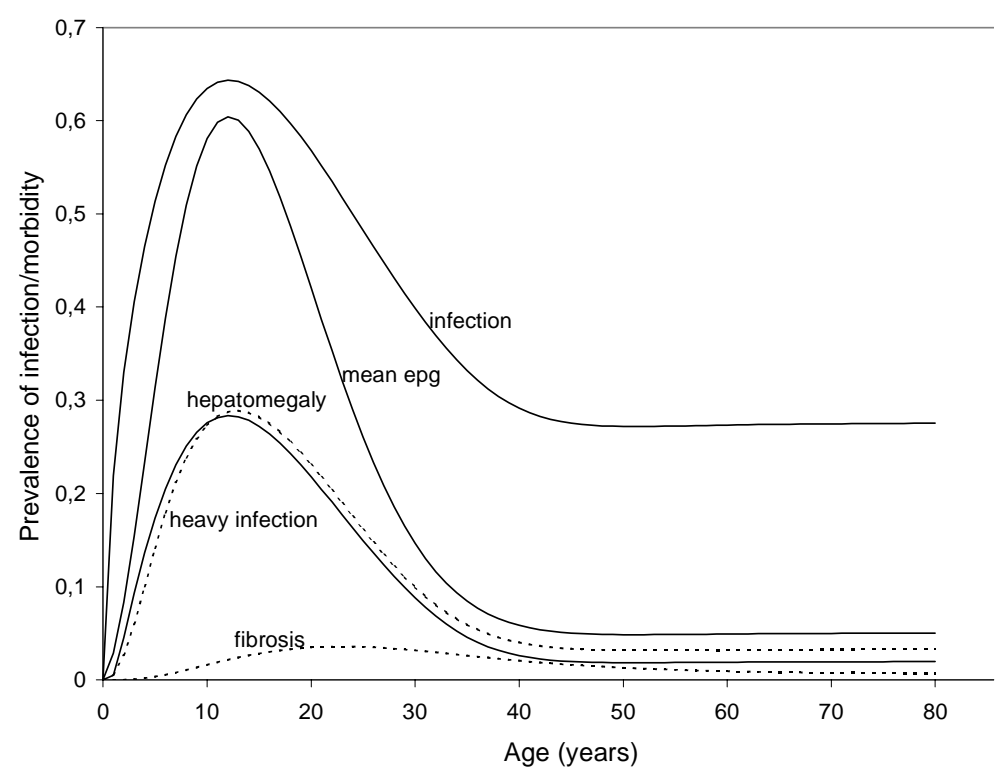

Fig. 2: age profiles of Schistosoma mansoni mean egg count and prevalence of infection and morbidity in the absence of treatment, based on the parameter values in Table I. The solid lines represent infection, and the dashed lines morbidity.

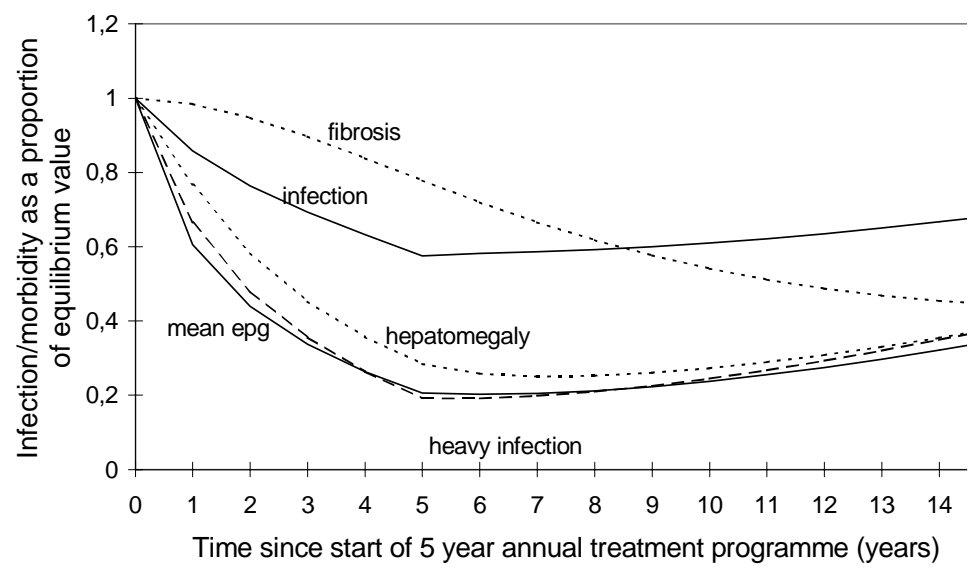

Fig. 3: the changes in infection and morbidity over time as a result of yearly treatment of 6-15 year olds for 5 years with a drug of $90 \%$ efficacy, and a coverage of $80 \%$. Levels of infection and morbidity are presented as a proportion of the equilibrium value.

instance, effectiveness is calculated for each year as the difference between the number of cases observed and the number of cases in the absence of treatment. Fig. 4 shows the age-profile for heavy infection at equilibrium and one year after treatment. The difference between these two lines represents the cases prevented. The figure also demonstrates that although only 6-15 years olds were treated, one year after treatment the reduction in heavy infection is observed not only in 7-16 year olds (target group is 1 year older), but also in the rest of the population. This reduction in the untreated group becomes even more evident as time goes on, and transmission levels are further reduced. Fig. 5 illustrates the changes in the prevalence of heavy infection with age with five years of yearly treatment, and after five years since the last treatment. The reduction in the prevalence of heavy infection extends well outside the age-groups which have been targeted for control. The difference between the equilibrium value and that observed in the presence of intervention is calculated for each year, weighted by the population size and summed over all years for which the programme is assessed to give the total number of case-years prevented.

If the total population is assumed to be 180,000 (see following section on programme framework), if there is no intervention then every year there will 


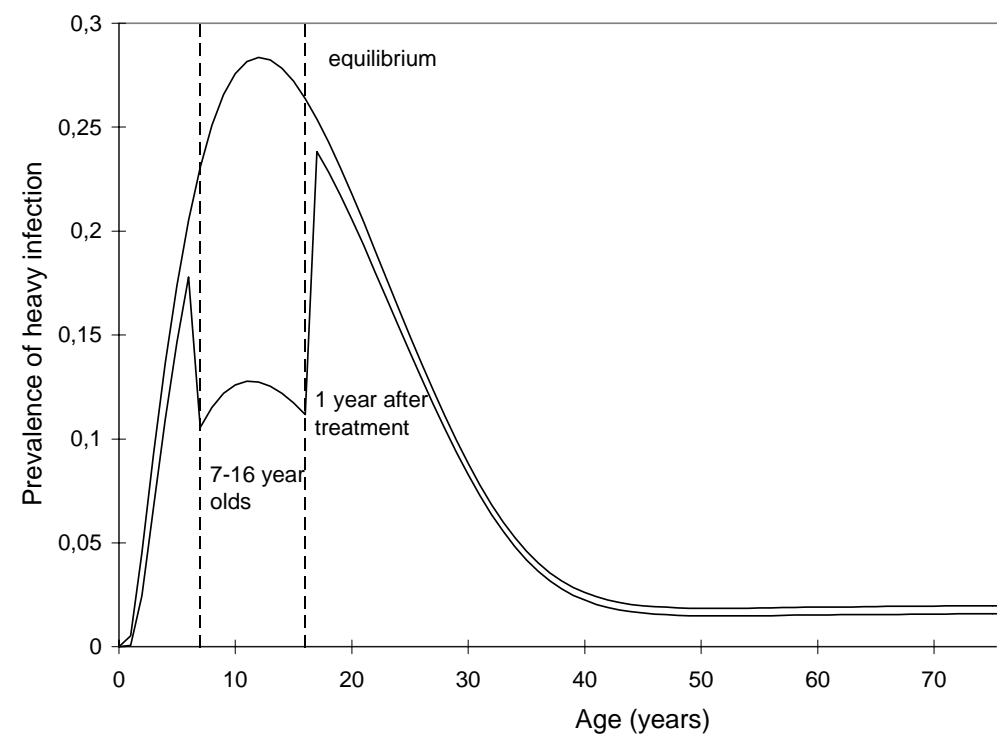

Fig. 4: comparison of the profile of prevalence of heavy infection with age at equilibrium and 1 year after treatment of 6-15 year olds with a drug of $90 \%$ efficacy and a coverage of $80 \%$.

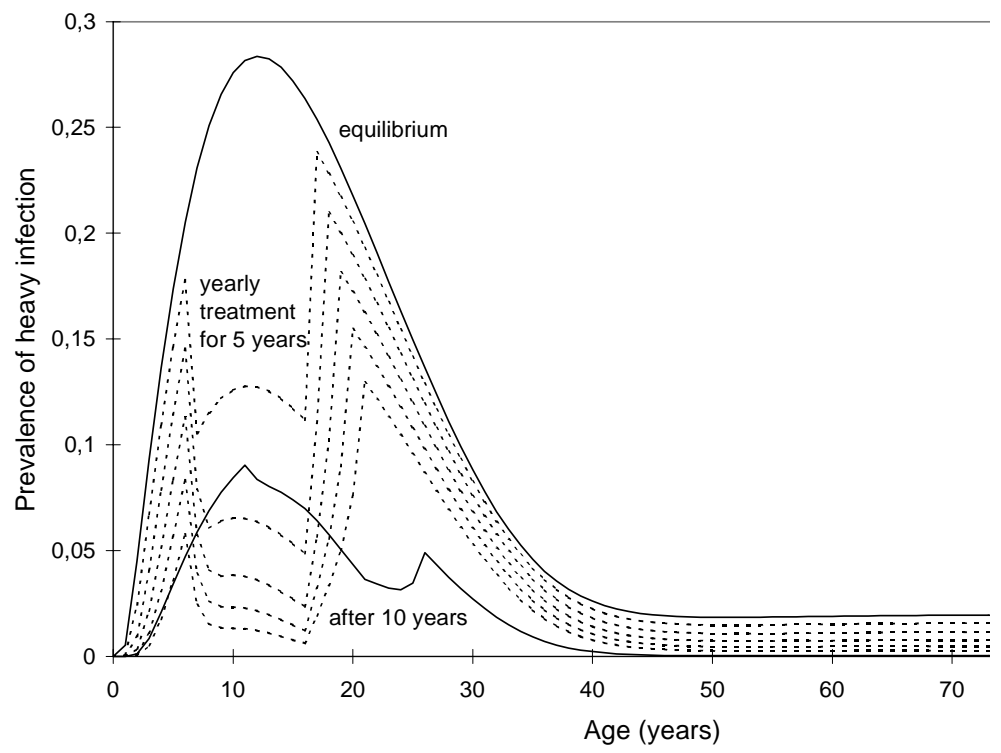

Fig. 5: the profiles of prevalence of heavy infection with age at equilibrium, and with 5 years of yearly treatment of 6-15 year olds with a drug of $90 \%$ efficacy and a coverage of $80 \%$. The dashed lines represents the levels of infection each year after each yearly treatment. The solid lines represent the levels at equilibrium and after 10 years since the first treatment.

be 105756 cases of infection, 48585 cases of heavy infection, 30341 cases of hepatomegaly and 3609 cases of fibrosis. Fig. 6 illustrates the number of case-years prevented (discounted at $5 \%$ ) when the effectiveness of five annual treatments is assessed after 5 years, 10 years and 15 years. Since the treatment programme has an impact on infection and morbidity that extends beyond the time frame of the treatment programme, the longer the time ho- rizon for evaluating effectiveness the greater the case-years prevented.

Programme framework- The framework for the delivery of praziquantel is based on a cost-effectiveness analysis of a school-targeted mass chemotherapy programme in the Kilombero District of Tanzania (Guyatt et al.1994). The programme is directed at all primary schools within the district. The drug is delivered to each school by a 


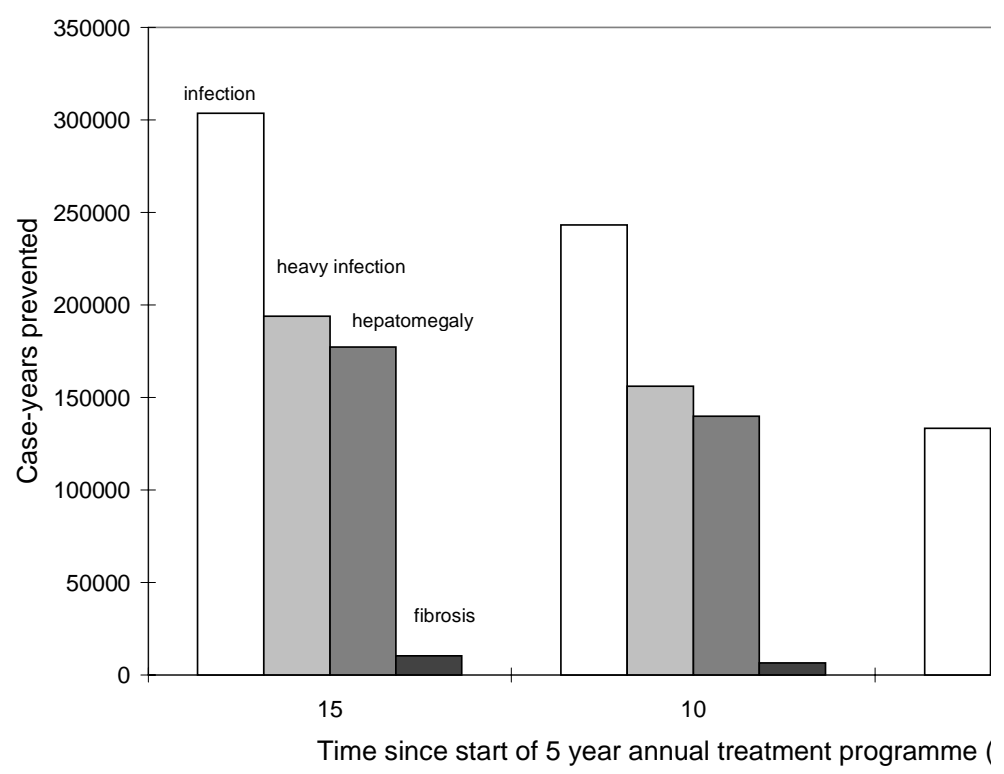

Fig. 6: the case-years prevented of infection, heavy infection, hepatomegaly and fibrosis at 15, 10 and 5 years since the start of the 5 year annual treatment programme of 6-15 year olds. Drug efficacy is $90 \%$ and coverage is $80 \%$. Effectiveness at 5 years corresponds to case-years prevented 1 year after the last treatment.

mobile team consisting of a driver, a fieldworker and a Rural Medical Aid. The unit cost menu is given in Table II, all costs given in 1997 prices (Ifakara Centre, Tanzania, pers. comm.).

The total population of the district is assumed to be 180,000 . Based on an standard age curve for Africa (population $\left.\mathrm{p}(\mathrm{a})=0.0455 . \mathrm{e}^{-(0.045 \mathrm{a})}\right)$, this implies that $28.6 \%(51,480)$ of these are in the agegroup 6-15. The number of children treated per day and the number of days taken in travelling the $1000 \mathrm{~km}$ to reach all schools are assumed fixed at 400 and 5 respectively. Programme coverage is the percentage of 6-15 year olds that are treated. Since not all 6-15 year olds attend school, coverage is the product of school attendance and patient com- pliance. Provider coverage is assumed to be $100 \%$ (all schools are visited by the mobile team). Although patient compliance is likely to be high, school attendance may be low. In this example, coverage is fixed at $80 \%$.

The effect of different frequencies of treatment The question of how often to treat is a central issue in the design of schistosomiasis control programmes. This analysis will consider the costeffectiveness of different frequencies of age-targeted treatment (6-15 year olds) using the model and cost framework described above. In each case, the model describes the implementation of a 5 year treatment programme. Four treatment strategies are compared, treating every 6 months, yearly, 2 yearly

TABLE II

Unit cost menu for the school-targeted treatment programme (costs in 1997 US\$)

\begin{tabular}{lllccc}
\hline & Input & Units & Unit cost & Quantities & $\begin{array}{l}\text { Cost for one } \\
\text { treatment cycle }\end{array}$ \\
\hline Personnel & Driver per diem & Per day & 20 & $108^{a}$ & 2160 \\
& Driver salary & Per day & 15.4 & $108^{a}$ & 1663.2 \\
& Fieldworker per diem & Per day & 20 & $108^{a}$ & 2160 \\
& Fieldworker salary & Per day & 9.8 & $108^{a}$ & 1058.4 \\
& Rural Medical Aid per diem & Per day & 20 & $108^{a}$ & 2160 \\
& Rural Medical Aid salary & Per day & 9.8 & $108^{a}$ & 1058.4 \\
Transport & Vehicle rental and running costs & Per km & 1.00 & 1000 & 1000 \\
Consumables & Praziquantel & Per dose & $0.583^{b}$ & $41184^{c}$ & 24010.27 \\
\hline
\end{tabular}

$a$ : number of days to treat children + number of days in travelling to schools; $b$ : Scientific Co-ordinating Centre for the Partnership for Child Development (pers. comm.); $c$ : number of children in target group * coverage. 
and 3 yearly. This corresponds to $10,5,3$ and 2 treatment cycles respectively. The drug efficacy is assumed to be $90 \%$. Effectiveness is assessed as case-years prevented over a 15 year time horizon, and both costs and effectiveness are discounted at a rate of 5\% (Drummond et al. 1987).

The relationship between effectiveness (caseyears prevented) and total programme cost resulting from different frequencies of treatment is illustrated in Fig. 7. Although the most effective option is to treat frequently (every six months), the most cost-effective option (in terms of minimising the cost-effectiveness ratio) is to treat infrequently (every three years) (see Table III). This is observed for all measures of effectiveness, although the actual cost per case-year prevented varies for each measure as a result of the differences in the number of case-years prevented (low for fibrosis, high for infection). Although three yearly treatment minimises the cost-effectiveness ratio, treating every two years, at an extra cost, provides an extra gain in effectiveness. The extra cost required to achieve an extra unit gain in effectiveness, by treating more frequently, is expressed in terms of incremental cost-effectiveness ratios, the extra cost per extra case-year prevented (Table IV). In pictorial terms, these values can be understood by examining the gradient of the line joining any of the two alternative strategies in Fig. 7. The steeper the line, the more efficient the more costly alternative, as any increase in cost returns a high increase in effectiveness. When the line becomes flatter, then a much larger investment yields only a small gain in effectiveness. As can be seen from Fig. 7, increasing the frequency of treatment has a greater impact on infection case-years prevented than fibrosis case-years prevented. The incremental costeffectiveness ratios also reveal diminishing mar-

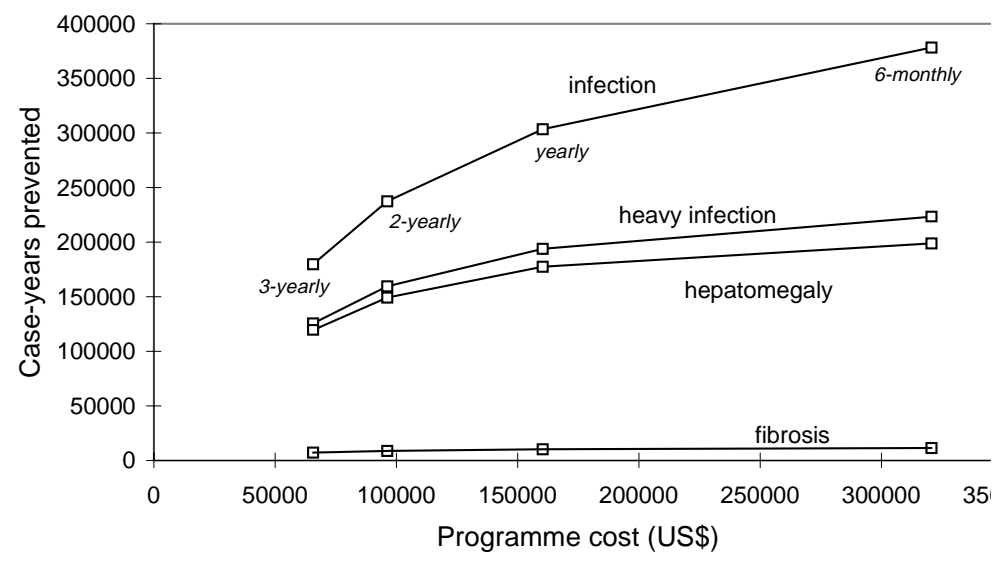

Fig. 7: the relationship between cost and case-years prevented with different frequencies of treatment. Treatment is targeted at 6-15 year olds over a 5 year period with a drug of $90 \%$ efficacy and a coverage of $80 \%$.

TABLE III

Cost-effectiveness ratios for different frequencies of treatment

\begin{tabular}{lllll}
\hline & 3 years & 2 years & 1 year & 6 months \\
\hline Cost per infection case-year prevented & 0.37 & 0.41 & 0.53 & 0.85 \\
Cost per heavy infection case-year prevented & 0.52 & 0.60 & 0.83 & 1.43 \\
Cost per hepatomegaly case-year prevented & 0.55 & 0.65 & 0.90 & 1.61 \\
Cost per fibrosis case-year prevented & 9.26 & 11.1 & 15.7 & 28.2 \\
\hline
\end{tabular}

TABLE IV

Incremental cost-effectiveness ratios for treating at a higher frequency

\begin{tabular}{lccc}
\hline $\begin{array}{l}\text { Extra cost required per extra } \\
\text { case-year prevented (US\$) }\end{array}$ & 2 yearly $v s 3$ years & yearly $v s$ yearly & 6 months $v s$ yearly \\
\hline Infection & 0.53 & 0.97 & 2.15 \\
Heavy infection & 0.90 & 1.86 & 5.43 \\
Hepatomegaly & 1.03 & 2.28 & 7.42 \\
Fibrosis & 19.7 & 40.1 & 142.7 \\
\hline
\end{tabular}


ginal returns i.e., as the frequency of treatment is increased a higher cost investment is required per extra gain in effectiveness if treating more frequently.

Although the cost per case-year prevented is crucial in identifying cost-effective strategies, budget constraints and minimum effectiveness targets are also important considerations. Budget constraints and minimum effectiveness targets may exclude options which although may be the most cost-effective, are not affordable or do not achieve certain goals in terms of effectiveness. In the above analysis, the most cost-effective strategy is the least expensive option, but the least effective, so may be excluded as a possible strategy if it does not achieve a given effectiveness target.

Interaction between drug price and drug efficacy - Praziquantel is the drug of choice for schistosomiasis (WHO 1993). There are now a large number of producers of generic praziquantel, and these different brands vary widely in price. The purchase price can vary between US $\$ 0.15$ to US\$ 0.60 per $600 \mathrm{mg}$ tablet for developing countries depending on the producer, purchaser and the amount procured (Scientific Co-ordinating Centre for the Partnership for Child Development, University of Oxford, pers. comm). Although there is some evidence that the bioavailability of these brands vary (Stierle 1994), there is no published quantitative data on the drug efficacy, in terms of reductions in levels in infection, of the more recent brands on the market. In the absence of data on the relative costs and efficacies of different brands of praziquantel it is difficult to make informed policy decisions on their usage.

The model by Chan et al. (1996a, b) and the cost framework described above has been used to investigate the interactions between drug efficacy and drug price in the implementation of a schooltargeted chemotherapy programme against $S$. mansoni (Guyatt \& Chan 1998). The analysis demonstrated that the interactions between drug efficacy and drug price were complex, where different combinations can result in similar cost-effectiveness (see Fig. 8). For example, a drug with an efficacy of $50 \%$ at a cost of US\$ 0.30 per dose would cost the same per case-year prevented as a drug with $90 \%$ efficacy at a cost of US\$ 0.70 per dose. The most important finding was the highly non-linear relationship between cost-effectiveness and drug efficacy, with low drug efficacies producing high and variable cost-effectiveness ratios. The analysis suggests that a drug which kills less than $50 \%$ of worms is not to be recommended. This has important practical implications for the widespread use of praziquantel since many of the international agencies procure praziquantel purely on the basis of price.

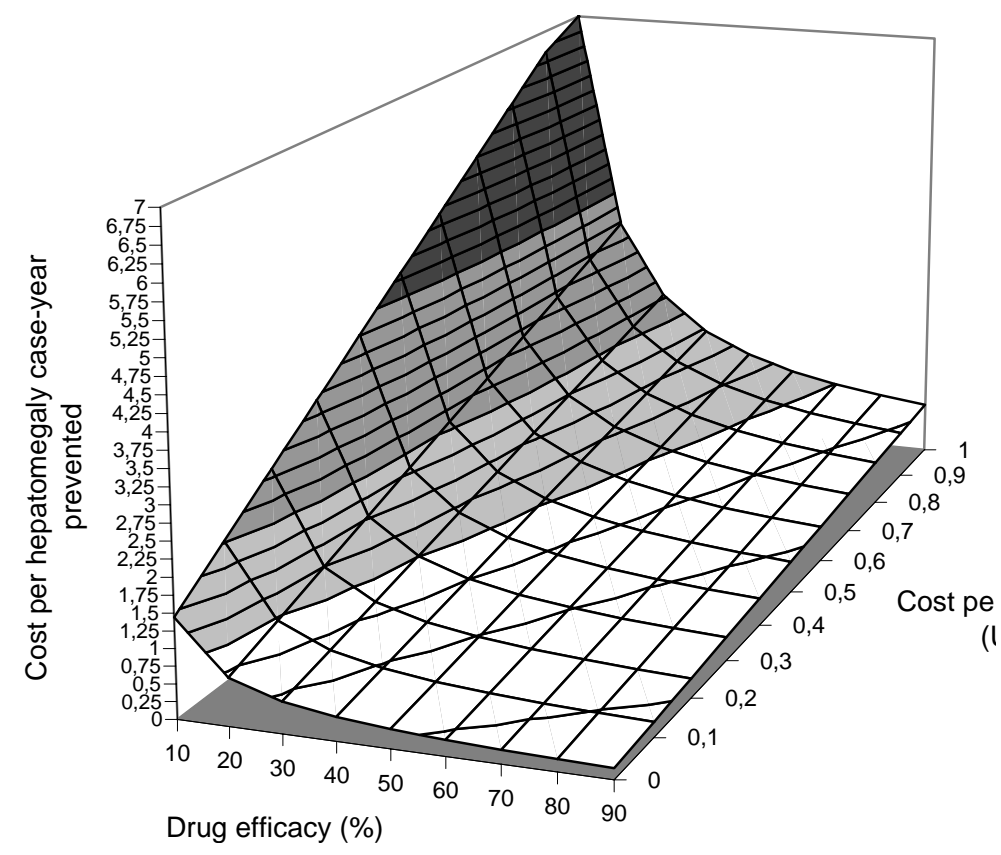

Fig. 8: the relationship between the cost per hepatomegaly case-year prevented and drug efficacy and drug price. The isoclines represent the combinations of drug efficacy and drug price which satisfy a given cost-effectiveness target. These are shown in steps of US\$ 0.25 per case-year prevented, and highlighted in shaded blocks for US\$ $0.00-1.00$, US\$ 1.00-2.00, US\$2.00-4.00, US\$ 4.00 and above. Based on analysis by Guyatt and Chan (1998), with $80 \%$ coverage. 
Sensitivity analysis - Sensitivity analysis of key variables is critical to undertaking a comprehensive cost-effectiveness analysis. The above analysis has demonstrated how important both drug price and drug efficacy are in affecting the cost-effectiveness of a treatment strategy. Other important variables would include coverage, other programme costs and levels of transmission. A full sensitivity analysis of these variables, is unfortunately beyond the scope of this paper, and as such the results presented here should be interpreted with caution. For instance, in the above analyses, coverage was fixed at $80 \%$. A decrease in coverage will reduce both the costs and the effectiveness of strategies, but not necessarily in proportion to one another. If the various frequencies of treatment were assessed at a lower coverage $(60 \%)$, although the most cost-effective option remains 3-yearly treatment, the cost per case-year prevented for each strategy varies, such that for frequent treatments the cost per case-year prevented is lower at $60 \%$, while for the less frequent treatments, they are lower at $80 \%$ coverage. At lower coverage, the incremental cost-effectiveness ratios for treating at higher frequencies are also smaller, suggesting that at lower coverages treating more frequently may be more favourable.

Composite measures of effectiveness - The above analysis considered four measures of effectiveness: case-years prevented for infection, heavy infection, hepatomegaly and fibrosis. Rather than considering each effectiveness measure separately, it may be more useful to consider the impact of all or some of the effectiveness measures collectively. This is clearly dependent on the objective of the control programme, with consideration of the potential consequences of each condition. The objective may be to reduce both hepatomegaly and fibrosis (clinical morbidity) or to reduce all morbidity, in which case the effectiveness would also need to consider heavy infection case-years prevented which may result in stunting and comprised educational and cognitive abilities. Constructing a composite outcome measure based on the caseyears of fibrosis, hepatomegaly, and heavy infection prevented is not trivial, but evidently needs to be explored. The 1993 World Development Report (World Bank 1993) has attempted this, albeit based on very different source data, and a recent analysis by Chan (1997) has used this framework to calculate composite measures of effectiveness for intestinal nematode infections. Clearly, some of the assumptions and methodologies used in calculating disability adjusted life years (DALYs) could be applied to explore the construction of a composite effectiveness measure for the model presented here for schistosomiasis, and this is the subject of future studies.

\section{ACKNOWLEDGEMENTS}

To Man-Suen Chan for useful discussions.

\section{REFERENCES}

Chan MS 1997. The global burden of intestinal nematode infections: 50 years on. Parasitol Today 13: 438443.

Chan MS, Bundy DAP 1997. Modelling the dynamic effects of community chemotherapy on patterns of morbidity due to Schistosoma mansoni. Trans $R$ Soc Trop Med Hyg 91: 216-220.

Chan MS, Anderson RM, Medley GF, Bundy DAP 1996b. Dynamic aspects of morbidity and acquired immunity in schistosomiasis control. Acta Tropica 62: 105-117.

Chan MS, Guyatt HL, Bundy DAP, Booth M, Fulford AJC, Medley GF 1995. The development and validation of an age structured model for schistosomiasis transmission dynamics and control. Epidemiol Infect 115: 325-344.

Chan MS, Guyatt HL, Bundy DAP, Medley GF 1996a. Dynamic models of schistosomiasis morbidity. Am J Trop Med Hyg 55: 52-62.

Drummond MF, Stoddard TL, Torrance GW 1987. Methods for the Economic Evaluation of Health Care Programmes, Oxford University Press, Oxford, 305 pp.

Guyatt HL, Chan MS 1998. An investigation into the interaction between drug efficacy and drug price of praziquantel in determining the cost-effectiveness of school-targeted treatment for Schistosoma mansoni using a population dynamic model. Trop Med Int Health 3: 425-435.

Guyatt HL, Evans D, Lengeler C, Tanner M 1994. Controlling schistosomiasis: the cost-effectiveness of alternative delivery strategies. Health Policy \& Planning 9: 385-395.

Prescott NM 1987. The economics of schistosomiasis chemotherapy. Parasitol Today 3: 21-25.

Prescott NM, Warford J 1985. Economic appraisal in the health sector, p. 127-145. In K Lee, A Mills (eds), The Economics of Health in Developing Countries, Oxford University Press, Oxford.

Rosenfield PL, Smith RA, Wolman MG 1977. Development and verification of a schistosomiasis transmission model. Am J Trop Med Hyg 26: 505-516.

Stierle F 1994. Effectiveness and Costs of Integrated Schistosomiasis Control: an Example of the Bandiagara District, Republic of Mali, MBA Thesis, School of Management and Health Economics, Keele University, 127 pp.

World Bank 1993. World Development Report: Investing in Health, Oxford University Press, Oxford, 329 pp.

WHO - World Health Organization 1993. The Control of Schistosomiasis. Second Report of an Expert Committee. Technical Report Series no. 830, Geneva, 86 pp. 